\title{
Past, current and future approaches to querying MAPK pathway activation: status and clinical implications
}

\begin{abstract}
MAPK pathway activation related to cancer development has drawn a great deal of attention in the field of personalized medicine in recent years. Many different approaches and assays have been developed to query the activation of this pathway and to develop life-saving treatments. The goal of this review article is threefold. First, to provide a brief overview of the many mutation assays that have been used to detect MAPK pathway activation, and to compare pros and cons of these assay platforms. Second, to focus on one custom-designed multiplexing mutation assay that is currently used to support an ongoing clinical trial and to show the novel features of this assay and its relevance in addressing unmet clinical needs. Third, to provide future perspectives of these MAPK pathway gene mutation detection efforts and to suggest how what we have learned from past and current approaches should guide future developments.
\end{abstract}

Keywords: BRAF $\bullet \mathrm{FFPE} \bullet \mathrm{KBN}-\mathrm{SNPE} \bullet \mathrm{KRAS} \bullet \mathrm{MAPK} \bullet \mathrm{MEK} \bullet \mathrm{NGS} \bullet \mathrm{NRAS} \bullet \mathrm{SNaPshot}$

\section{Background}

Defining the mutational status of solid tumors is becoming one of the most important personalized medicine approaches in guiding treatment options for cancer patients [1]. Treatments developed against targets within a specific signaling pathway may not be effective when activating mutations are downstream of the pathway. One well-known example is that tumors carrying activating mutations in RAS and RAF do not respond well to anti-EGFR therapy [2]. Inhibitors against proteins further downstream in the pathway, such as ERK, may be more effective [3]. Therefore, proteins that lie downstream of RAS, RAF and MEK in the RAS/MAPK pathway (Figure 1) are likely potential targets for anti-cancer agent development [4-6]. Approved and investigational treatments selective for MAPK pathway targets are listed in Table 1 [3,7-27].

\section{RAS, RAF mutation distributions} among different cancers

Approximately 25\% of all cancers carry RASactivating mutations [28]. In pancreatic cancer and colorectal cancer, these mutations, especially in KRAS, are present at even higher rates $[4,28]$. Roughly $10-25 \%$ of melanomas also carry NRAS mutations $[4,6,29]$ and KRAS mutations were detected in nearly $30 \%$ of non-small cell lung cancers $[4,30]$. For thyroid cancers, RAS mutations (HRAS, KRAS, or NRAS) represent $55-60 \%$ of cases, and BRAF mutations were found in nearly $60 \%$ of malignant melanomas [6,31,32]. These BRAF mutations are mainly located in the kinase domain, and a single base substitution (V600E) accounts for $-80 \%$ of mutations $[6,31,32]$. Not all activating BRAF mutations are found in melanoma. Approximately 10\% of colorectal cancer tumors harbor BRAF mutations and approximately $50 \%$ of thyroid cancers carry BRAF mutations [29], as does several percent of non-small-cell lung cancer cases [33]. The high frequency of RAS or RAF mutations in these cancers makes targeting this pathway an attractive strategy for new anti-cancer agent development for those individuals most likely to benefit from MAPK inhibitors [34]. The percentage of selected

\section{Ken CN Chang *,1}

\& Matthew J Marton ${ }^{1}$ ${ }^{1}$ Molecular Biomarkers \& Diagnostics, Merck \& Co, Inc., Rahway, NJ, USA *Author for correspondence: ken.chang@merck.com
Future
Medicine
part of fss 


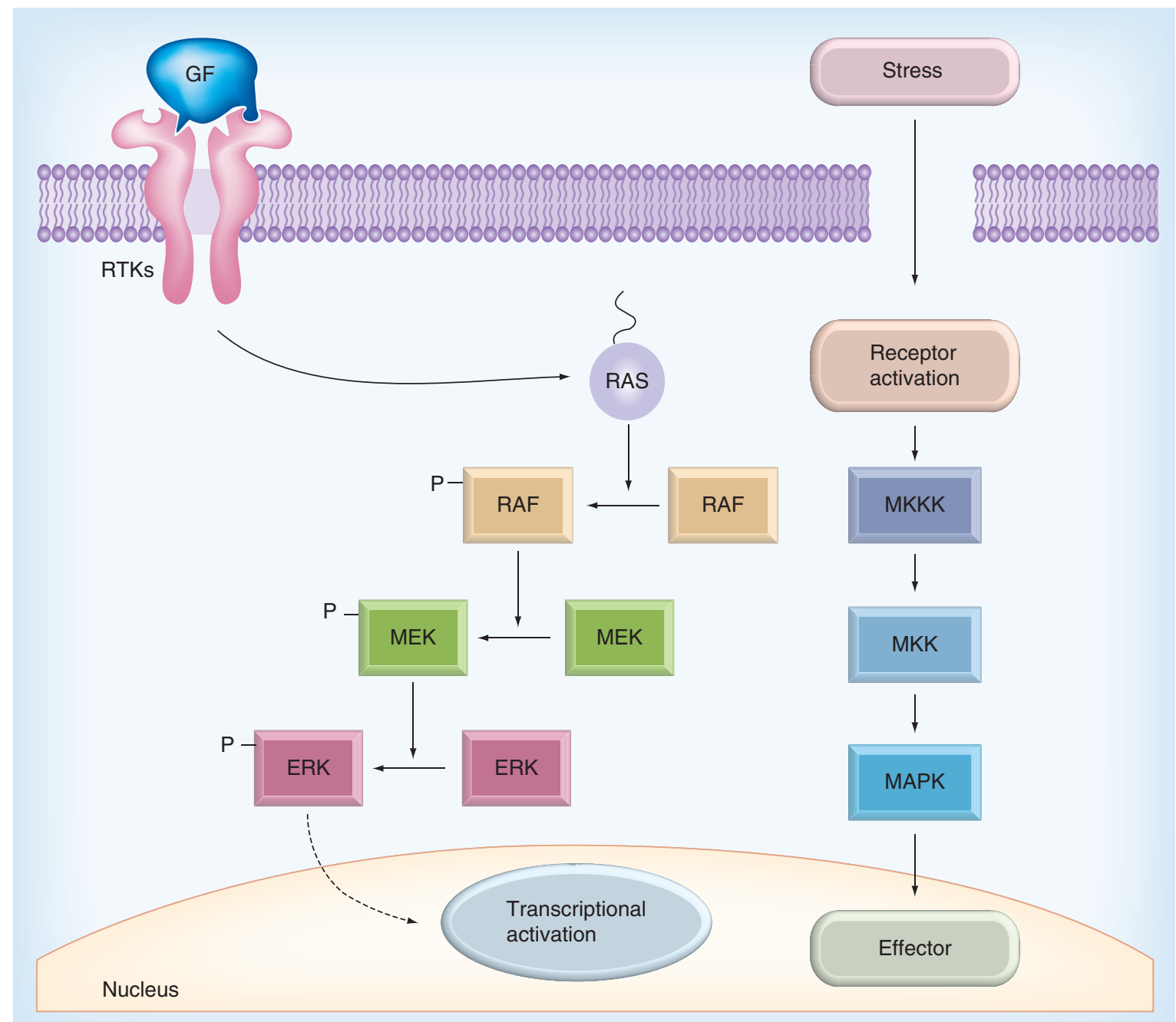

Figure 1. MAPK pathway. GF: Growth factor.

tumors with mutations in KRAS, BRAF or NRAS as reported in the COSMIC database is summarized in Table 2. Other MAPK pathway genes such as ERK and MEK are also targets for pathway intervention; however, they are not oncogenes, and therefore strategies to target these proteins must rely on pathway activation assays other than those in ERK or MEK, since mutations in these genes cannot be used as a diagnostic tool to identify a responsive patient population.

\section{Mutations \& clinical outcomes}

Hotspot mutations in these MAPK oncogenes, such as those located in KRAS codons 12, 13 and 61, and BRAF 600 , have long been the primary scientific and clinical focus. Increasingly, however, recent evidence indicates mutations in other locations can also lead to the tumorigenic phenotype $[35,36]$ and that it is important to detect mutations beyond those in well-known hotspots. For example, a recent clinical trial revealed panitumumab (Vectibix), an anti-EGFR monoclonal antibody $(\mathrm{mAb})$, was beneficial in patients with wildtype KRAS or NRAS but potentially harmful in patients with KRAS or NRAS mutations that are not routinely covered in regular tests [37]. Using KRAS as an example, not all mutations have the same clinical outcome, and often rarer mutations detected outside of the frequently encountered codons 12 and 13 are mutually exclusive making them an important category of mutations with diagnostic value [38]. Furthermore, approximately $1-10 \%$ of KRAS mutated tumors responded to anti-EGFR mAb therapy [39]. Retrospective analysis suggested that patients who received $\mathrm{mAb}$ therapy with KRAS G13D mutation had better outcomes than those with other KRAS mutations [39]. In vitro analysis also supported the hypothesis that not all KRAS mutations have the same biological impact [40]. Therefore, it may be necessary to include a more extensive list of known MAPK pathway gene 
Table 1. Approved and investigational drugs selective for MAPK pathway targets.

\begin{tabular}{|lllr|}
\hline MAPK target & Tumor & Treatment options or investigational drugs & Ref. \\
KRAS, HRAS & Melanoma, NSCLC & BMS-214662, L-778123 & {$[7-11]$} \\
\hline MEK & Breast, lung & Cl-1040 & {$[12]$} \\
MEK & NSCLC, melanoma & PD-0325901 & {$[13,14]$} \\
MEK & Pancreatic, thyroid & BAY 86-9766 & {$[15]$} \\
MEK & Advanced malignancies & AZD8330 & {$[16]$} \\
BRAF & Melanoma & Dabrafenib & {$[17]$} \\
MEK/BRAF & Melanoma & Trametinib & {$[18]$} \\
BRAF & Melanoma & Venmurafenib & {$[19,20]$} \\
BRAF & NSCLC, leukemia, and so on & BAY 43-9006 & {$[21,22]$} \\
BRAF & Melanoma & PLX4720 & {$[23,24]$} \\
RAF & NSCLC, CRC, and so on & XL281 & {$[25]$} \\
BRAF & Melanoma & GSK2118436 & {$[26,27]$} \\
ERK & Melanoma, CRC & MK-8353 & {$[3]$} \\
\hline CRC: Colorectal cancer; NSCLC: Non-Small-cell lung cancer. & & \\
\hline
\end{tabular}

mutations in future routine testing. The goals of this review are: to provide a brief overview of many mutation assays that could be or have been used to detect MAPK pathway gene activation and also compare pros and cons of these assay platforms; to focus on one custom-designed multiplexing mutation assay that is currently used to support an ongoing clinical trial and to show the novel features of this assay and its relevance in addressing unmet clinical needs; and to provide our perspective on future MAPK pathway gene mutation detection efforts and to suggest how best to apply what we have learned from past and current approaches to guide future developments.

\section{Brief overview of mutation assays that detect MAPK pathway gene activation}

In general, one can classify the type of MAPK pathway activation mutation assays into two broad categories: those that detect mutations in specific, limited nucleotide locations, and those that take a general sequencing approach such as Sanger sequencing or pyrosequencing. If the location of the mutations is known, there are many more assay platforms from which to select, each having its own advantages and disadvantages.

Sanger sequencing, or dideoxy sequencing, is still considered the 'gold standard' for mutation detection, even though next-generation sequencing (NGS) may someday replace it as the new standard [41]. The dideoxy-sequencing methodology became widespread in the 1990s when Applied Biosystems (ABI)'s capillary electrophoresis DNA sequencers were used ubiquitously to first sequence the human genome. It is well accepted and understood, in part because base-calling is automated and because no complex bioinformatics are needed to analyze the raw data, as the electropherograms, with color-coordinated trace peaks corresponding to each base, can be unambiguously visualized. However the amount of input DNA needed is usually relatively large, especially if longer sequences need to be analyzed. The use of clinical formalin-fixed paraffin embedded (FFPE) tissue samples, which normally are highly fragmented, also adds to the requirement of higher input DNA to generate clear-cut data. Another challenge is that all different alleles within the same amplicon are sequenced at the same time using the same set of sequencing primers, therefore it is very difficult to distinguish if two mutations are present in one allele in the same amplicon region or one mutation is present in two alleles.

Pyrosequencing can be considered the original 'sequencing-by-synthesis' technology. As DNA is synthesized from an immobilized template, the released pyrophosphate triggers the sulfrylase-luciferase cascade

Table 2. Percentage of selected tumors with mutations in KRAS, BRAF or NRAS as reported in the COSMIC Database v68.

\begin{tabular}{|llll|}
\hline & \multicolumn{3}{c|}{ Cosmic } \\
\hline KRAS & BRAF & NRAS \\
\hline Melorectal & 35 & 13 & 4 \\
\hline Ovarian & 2 & 42 & 15 \\
\hline Pancreatic & 12 & 8 & 1 \\
\hline Thyroid & 57 & 2 & 1 \\
\hline Lung & 2 & 39 & 6 \\
\hline
\end{tabular}


resulting in the emission of light [42]. Nucleotides are added one at a time in order, and the emission of light and the amount of emission allow the determination of which nucleotide base and the number of nucleotides that is incorporated. Since the amount of light emitted is proportional to the number of nucleotides incorporated it is possible to identify different alleles and to determine the variant frequencies.

Many technologies have been developed to detect mutations of known locations, but they differ by degree of sensitivity, sample requirements and the suitability of multiplexing. The $\mathrm{SNaPshot}$ multiplex kit is designed to interrogate SNPs at known locations on DNA templates [43-45]. Genomic DNA is isolated from FFPE samples and specific exons from different target genes are amplified in one or more multiplexed PCR reactions. These PCR products then act as templates in the $\mathrm{SNaPshot}$ reaction. This reaction consists of oligonucleotide probes designed to hybridize immediately adjacent to the $3^{\prime}$ side of the nucleotides of interest. The probe is then extended by the addition of a single fluorescently-labeled dideoxynucleotide. Detection of mutations is made by a combination of the identification of the fluorescently tagged nucleotide added and the migration of the probe, based on its size as determined by capillary electrophoresis.

The design strategy of shifted termination assay (e.g., ABI, TrimGen) is similar to that of the $\mathrm{SNaPshot}$ assay in that it utilizes a primer with complementary sequence up to the base 3 ' to the target nucleotide site. This is followed by supplying the mutant nucleotide with respective fluorescently-labeled ddNTP to allow early termination of chain extension if a variant or mutation is present [46]. The resulting wild-type fragment will be larger than the mutant fragment, therefore allowing separation using electrophoresis.

The StripAssay ${ }^{\mathrm{TM}}$ [47] from ViennaLab (Vienna, Austria) is a mutation assay technology in which biotin-labeled PCR amplicons of target genes are hybridized to a solid matrix with up to 48 immobilized probes complementary to the target DNA. Since the probes are labeled with biotin, streptavidin-alkaline phosphatase incubation allows color to be observed and the assay result to be observed and scored. Although assays for several genes are available, adding assays for additional mutations may be cumbersome because each would have to be designed separately, that is, the assay does not appear to be easily scalable. In addition, the assay does not permit quantification of the mutant allele frequency, in other words, percent mutant for each mutation in the corresponding samples can only be estimated, as only 'present' or 'absent' results are obtained. This type of mutation detection has a very specific purpose and may not be applicable for broad applications since treatment responses may be directly related to the variant frequency of a particular mutation, and retrospective analysis of such information may lead to hypotheses to better predict patient treatment response.

Several qualitative allele-specific PCR technologies, such as the Amplification Refractory Mutation System (ARMS, Qiagen), achieve high sensitivity by blocking amplification of the wild-type sequence [48]. ARMS is a mutation assay that employs so-called Scorpion ${ }^{\mathrm{TM}}$ probes that, under ambient temperature, will form a hair-pin loop with a fluorescent tag that is quenched at one end and a target primer sequence attached at the other end [48]. After target amplification through the primer end, heat denaturation allows the newly synthesized strand to be opened up together with the hair-pin loop. During the cooling step, this hair-pin loop with a predesigned sequence complement hybridizes to the newly synthesized target sequence. Since the hybridization between the newly synthesized sequence and the complementary sequence within the loop will keep the quencher separated from the dye, fluorescent signal will be emitted. Kinetic analysis of the fluorescent signals and delta Ct calculation allow the determination of the presence of potential mutations.

TaqMan ${ }^{\mathrm{TM}}$ SNP Genotyping Assay (ABI) is one of the most well-established variant detection (or allelic discrimination) assays [49]. Thousands of known single nucleotide variant assays can be ordered directly from $\mathrm{ABI}$, many of which have been prevalidated by the company. Assays for novel mutations can be easily custom designed and ordered. The design principle is basically a Taqman ${ }^{\mathrm{TM}}$ assay with fluorescently-quenched probe designed to hybridize to a region including nucleotide of the mutation of interest. If the mutation is present, the probe is degraded by the 5' exonuclease activity of the Taq polymerase, which releases the quencher from the probe and allows its fluorescence to be detected. Using different primer-probe sets and different fluorophores for mutant and wild-type probes allows both alleles to be amplified in the same reaction and permits quantification of the relative abundance of the mutant and wild-type alleles.

OncoCarta Mass Array (Sequenom) is a mass spectrometry-based (MALDI-TOF) mutation assay based on a single-nucleotide primer extension (SNPE) strategy [50]. A panel of predesigned mutations (up to several hundred mutations) can be assembled; however, the bigger the panel the more DNA is required. Detection sensitivity for this assay is not very high, and some of the pros and cons of these assays can be found in Table 3.

Other mutation detection assays are also available if qualitative analysis of presence or absence of mutations is a sufficient result. These assays include a melting 
Table 3. Pros and cons of the various platforms suitable for MAPK activation detection assays.

\begin{tabular}{|c|c|c|}
\hline Platform & Pros & Cons (or limitations) \\
\hline Sanger sequencing & $\begin{array}{l}\text { - Foreknowledge of mutation not } \\
\text { needed } \\
\text { - Current gold standard assay } \\
\text { - Able to detect any mutation including } \\
\text { in/dels }\end{array}$ & - Low sensitivity (10-20\%) \\
\hline Pyro-sequencing (Qiagen) & $\begin{array}{l}\text { - High sensitivity }(1-5 \%) \\
\text { - Able to detect any mutation including } \\
\text { in/dels } \\
\text { - Commercial kit for KRAS codons } 12,13 \\
\text { and } 61\end{array}$ & $\begin{array}{l}\text { - Potential false positives } \\
\text { - Higher costs than Sanger } \\
\text { sequencing }\end{array}$ \\
\hline SNaPshot (ABI) & $\begin{array}{l}\text { - High sensitivity }(1 \%) \\
\text { - Easily configurable to add mutations of } \\
\text { interest }\end{array}$ & $\begin{array}{l}\text { Detects user-defined } \\
\text { mutations }\end{array}$ \\
\hline STA (ABI) & $\begin{array}{l}\text { - High sensitivity ( } 1 \%) \\
\text { - Commercial kit available for KRAS } \\
\text { codons } 12 \text { and } 13\end{array}$ & $\begin{array}{l}\text { Detects KRAS: Gly12/13 } \\
\text { (Ser, Arg, Cys, Asp, Ala, Val) } \\
\text { BRAF: Val600 (Glu, Ala, Gly) }\end{array}$ \\
\hline STA (TrimGen) & $\begin{array}{l}\text { - High sensitivity ( } 1 \%) \\
\text { - Commercial kit available for KRAS } \\
\text { codons } 12 \text { and } 13\end{array}$ & $\begin{array}{l}\text { Detects KRAS: Gly12Ser, } \\
\text { Gly12/13 (Arg, Cys, Asp, } \\
\text { Ala, Val) }\end{array}$ \\
\hline StripAssay (Vienna Labs) & $\begin{array}{l}\text { - High sensitivity ( } 1 \% \text { ) } \\
\text { - Commercial kit available for codons } 12 \\
\text { and } 13\end{array}$ & $\begin{array}{l}\text { Detects KRAS: Gly12Ala, Arg, } \\
\text { Ile, Leu, Ser, Val, Gly12/13 } \\
\text { (Asp, Cys) } \\
\text { - BRAF: Val600Glu }\end{array}$ \\
\hline ARMS \& Scorpions (Qiagen) & $\begin{array}{l}\text { - EU approved; US FDA approved as IVD } \\
\text { (Qiagen); } \\
\text { - RUO kit available in the USA } \\
\text { - High sensitivity }(1 \%)\end{array}$ & $\begin{array}{l}\text { Detects KRAS Gly12Ala, Arg, } \\
\text { Cys, Ser, Val, Gly12/13Asp }\end{array}$ \\
\hline TaqMan (ABI) & - Many off-the-shelf assays available & $\begin{array}{l}\text { Detects user-defined } \\
\text { mutations }\end{array}$ \\
\hline MALDI-TO (Sequenom) & $\begin{array}{l}\text { Large number of hotspot mutations } \\
\text { in many genes are analyzed in a single } \\
\text { assay }\end{array}$ & - Low sensitivity $(10 \%)$ \\
\hline MeltingCurve (Roche) & $\begin{array}{l}\text { - EU approved; FDA approved as IVD } \\
\text { (cobas) }\end{array}$ & $\begin{array}{l}\text { - Assay output is 'mutant' or } \\
\text { 'mutation not detected' }\end{array}$ \\
\hline Surveyor (Transgenomic) & $\begin{array}{l}\text { - High sensitivity }(1 \%) \\
\text { - Commercial kit available for KRAS } \\
\text { codons } 12,13 \text { and } 61\end{array}$ & $\begin{array}{l}\text { - Assay output is 'mutant' or } \\
\text { 'mutation not detected' }\end{array}$ \\
\hline
\end{tabular}

curve assay like those Roche cobas ${ }^{\mathrm{TM}}$ assays [51,52] and the hybridization nuclease digestion assays such as Surveyor ${ }^{\circledR} K R A S$ Mutation Kit from Transgenomic [53].

Commercially available US-FDA-reviewed KRAS and $B R A F$ kits, such as the Qiagen Therascreen ${ }^{\circledR} K R A S$ RGQ kit and the cobas $K R A S$ and $B R A F$ mutation tests, detect mutations only at limited locations and do not include an extended set of mutations that might be critical to treatment response. The Therascreen KRAS assay is based on Scorpions and ARMS technologies and detects seven frequently encountered mutations in codon 12 and 13 [54]. The cobas KRAS mutation kit is a real-time PCR assay which detects 21 mutations in codons 12, 13 and 61 [51]. However, specific mutations are not reported, which is important for exploratory data analysis. The cobas $B R A F$ assay only detects $B R A F$ V600E mutation [52]. In the bioMerieux $\mathrm{THxID}^{\mathrm{TM}}$ $B R A F$ kit, which uses the ARMS methodology, two different probes labeled with two different dyes allow the simultaneous detection of the $B R A F$ internal control and a $B R A F$ mutation [55]. In the THxID assay, primers specific for the $B R A F$ gene allow the amplification of a non-polymorphic gene area, which is used as an internal control. Primers specific for the mutations 
V600E and V600K allow the amplification of mutated fragments, and hence, BRAF mutation detection. Although many mutation assays are considered semiquantitative assays, no FDA-regulated clinical assays currently report the mutation variant frequency (VF), partially due to the difficulty of interpreting the clinical meaning of VF, as well as the reproducibility of these semi-quantitative measures.

\section{Clinical consideration, clinical objectives $\&$ assay platform requirements}

Based on the clinical objectives, many clinical considerations are important to the selection of a specific assay for the clinical application. Table 3 summarizes the major pros and cons of each platform mentioned above and can be used as a reference when considering platform selection options. Other approaches for querying MPAK pathway include identification of MAPK gene expression signature [56], however it is not the intention to review this strategy here.

Additional assay development considerations include tissue types, sample collection and preservation methods, clinical objectives, turn-around time (TAT), and acceptable false positive/false negative rates [57]. For tissue types, FFPE tissue is one of the most unique and challenging since nucleic acids isolated from there are typically highly fragmented. Many assay designs involve PCR amplification of target amplicons and if the length of target amplicon is too long (such as greater than $150 \mathrm{bp}$ ) there will be a very limited number of copies of the target sequences available for PCR amplification, which can introduce large variation simply due to the sampling variation. Sample collection and preservation methods will also likely impact the quality of nucleic acids, since fragmentation and degradation can affect data variation and reproducibility. In the clinical setting, including clinical trials, a fast TAT is always a goal as patients may not be able to wait for 2 weeks for data to be available, and a clinical decision can be made. In our experience, the normal expectation of TAT is 7 calendar days or 5 business days. Finally, a good balance of low false-positive and low false-negative rates is also the key to making an assay 'the platform of choice'. The following custom-designed multiplexing mutation assay, which was developed and analytically validated in our lab, took most of the above-mentioned key factors into consideration and is currently being used with great success to support an ongoing clinical trial.

\section{Novel features of a custom-designed multiplexed mutation assay (KRAS, BRAF, NRAS SNPE mutation detection assay)}

The KRAS, BRAF, NRAS SNPE (KBN-SNPE) mutation detection assay was designed to support an ongoing clinical trial for patient prospective enrollment [58]. The assay plan and design started with identifying 35 mutations in the KRAS/BRAF/NRAS genes that are thought to activate the MAPK pathway or increase activity of one of the kinases [59-64]. A custom-designed SNPE called the KBN-SNPE assay, was then developed using $\mathrm{SNaPshot}$ mutation assay kits from ABI. The SNaPshot methodology takes advantage of PCR-based amplification of the target sequence followed by specific primers/probes to interrogate the mutation sites of interest through single nucleotide extension reactions using fluorescently labeled ddNTP and capillary gel electrophoresis to separate the resulting products. Similar assays were published in several different research studies and some were for clinical sample analysis $[60,61,65]$; however, none of these assays were designed and validated to support a clinical trial for patient prospective enrollment.

This assay begins with the PCR amplification of regions of the KRAS, BRAF and NRAS genes from gDNA isolated from one or two $5 \mu \mathrm{m}$ FFPE tissue slides. A set of custom-designed probes with different lengths of tails with repeating sequences is hybridized to the PCR amplicons, followed by a single base extension reaction of the probes. The final products are then separated by the capillary electrophoresis, and GeneMapper software (ABI) is used to make automated base calls based on size and incorporated fluorescent label. An example of a KBN-SNPE assay output is shown in Figure 1.

This KBN-SNPE assay was designed to detect the mutations of interest in KRAS, BRAF and NRAS (Table 4) in support of patient stratification in clinical trials. The design and validation of KBN-SNPE assay took into the consideration of the challenge of FFPE samples, which generally yield fragmented, low quality genomic DNA. Based on our clinical experience, the average fragment size of FFPE tissue gDNA is around 200 base pair [45]. Therefore, it is necessary to design PCR primers that amplify short stretches (100$150 \mathrm{bp}$ ) of DNA and to balance the primer concentrations so that all target regions within same reaction can be equally amplified.

The KBN-SNPE assay concurrently interrogates nine and six nucleotide positions within KRAS and $N R A S$, respectively, and twelve within $B R A F$ (Table 4). Hot spot mutation regions of $K R A S$ exons 2, 3 and 4, NRAS exons 2 and 3, and BRAF exons 11 and 15 were amplified in three separate PCR reactions. Due to the number of SNPE probes used for $B R A F$ and sequence similarity between $K R A S$ and $N R A S$ plus a potential need for accommodating newly discovered critical mutations while the clinical trial progresses, it was necessary to perform the primer extension portion 
Table 4. Mutations identified by the KBN-SNPE assay and their resulting amino acid change.

\begin{tabular}{|c|c|c|c|c|c|c|c|c|c|c|c|}
\hline \multicolumn{4}{|c|}{ BRAF } & \multicolumn{4}{|c|}{ KRAS } & \multicolumn{4}{|c|}{ NRAS } \\
\hline $\begin{array}{l}\text { Codon } \\
\text { number }\end{array}$ & $\begin{array}{l}\text { WT } \\
\text { codon }\end{array}$ & $\begin{array}{l}\text { Mutant } \\
\text { codon }\end{array}$ & $\begin{array}{l}\text { Protein } \\
\text { description }\end{array}$ & $\begin{array}{l}\text { Codon } \\
\text { number }\end{array}$ & $\begin{array}{l}\text { WT } \\
\text { codon }\end{array}$ & $\begin{array}{l}\text { Mutant } \\
\text { codon }\end{array}$ & $\begin{array}{l}\text { Protein } \\
\text { description }\end{array}$ & $\begin{array}{l}\text { Codon } \\
\text { number }\end{array}$ & $\begin{array}{l}\text { WT } \\
\text { codon }\end{array}$ & $\begin{array}{l}\text { Mutant } \\
\text { codon }\end{array}$ & $\begin{array}{l}\text { Protein } \\
\text { description }\end{array}$ \\
\hline 466 & GGA & GAA & G466E & 12 & GGT & AGT & G12S & 12 & GGT & GAT & G12D \\
\hline 466 & GGA & GTA & G466V & 12 & GGT & TGT & $\mathrm{G} 12 \mathrm{C}$ & 12 & GGT & GTT & G12V \\
\hline 592 & ATA & GTA & I592V & 12 & GGT & GAT & G12D & 13 & GGT & CGT & G13R \\
\hline 594 & GAT & GTT & D594V & 12 & GGT & $\mathrm{GCT}$ & G12A & 13 & GGT & GAT & G13D \\
\hline 594 & GAT & GAA & D594E & 12 & GGT & GTT & G12V & 61 & CAA & AAA & Q61K \\
\hline 594 & GAT & GAG & D594E & 13 & GGC & CGC & G13R & 61 & CAA & CGA & Q61R \\
\hline 596 & GGT & CGT & G596R & 13 & $\mathrm{GGC}$ & GAC & G13D & 61 & CAA & CTA & Q61L \\
\hline 597 & CTA & TCA & L597S & 61 & CAA & AAA & Q61K & 61 & CAA & CAC & Q61H \\
\hline 600 & GTG & GAG & V600E & 61 & CAA & CTA & Q61L & 61 & CAA & CAT & Q61H \\
\hline 600 & GTG & AGG & V600R & 61 & CAA & CAC & Q61H & & & & \\
\hline 600 & GTG & AAG & V600K & 61 & CAA & CAT & Q61H & & & & \\
\hline 600 & GTG & GAT & V600D & 146 & GCA & ACA & $\mathrm{A} 146 \mathrm{~T}$ & & & & \\
\hline 601 & AAA & GAA & K601E & 146 & GCA & GTA & $\mathrm{A} 146 \mathrm{~T}$ & & & & \\
\hline
\end{tabular}

of the assay in three separate reactions [45]. Collectively, this assay can detect 35 specific mutations (81 potential mutations for all possible $\mathrm{A} / \mathrm{T} / \mathrm{G} / \mathrm{C}$ changes at each nucleotide location excluding wild-type) at 27 nucleotide positions in three genes spread over seven exons.

The analytical validation process of KBN-SNPE assay involved a triplicate strategy and relied on several novel approaches to redefine and calculate sensitivity, specificity and accuracy. Some highlights of this novel assay validation strategy are captured in the following sections.

\section{Calculation of specificity, sensitivity \\ \& concordance}

For accuracy study, concordance analysis was done at the sample (patient) level. Each sample was subjected to mutation analysis by both KBN-SNPE assay and Sanger sequencing. If the two methods agreed, the result was assumed to be accurate. If only one method detected a mutation, the sample was analyzed by Ion Torrent Ion PGM sequencing. If the Ion PGM result matched either the KBN-SNPE or Sanger result, the matched result was considered accurate.

This concordance approach using next-generation sequencing to confirm the Sanger sequencing result (considered as a gold-standard sequencing assay) is somewhat unconventional since NGS is a more sensitive sequencing assay and some mutations detected by the KBN-SNPE assay may not be detected using the
Sanger sequencing. The following terms were defined in advance in order to carry out the analytical validation for this multi-gene/multi-mutation site validation and to set up the acceptance criteria:

Specificity or sample-level negative agreement:

- Specificity $=$ \# of True negatives $/$ \# of True negatives + \# of False positives), where the truth is defined as the consensus result obtained by two or more assay methods (KBN-SNPE, Sanger sequencing, NGS)

Sensitivity or sample-level positive agreement:

- Sensitivity = \# of True positives $/$ (\# of True positives + \# of False negatives)

\section{Overall concordance (accuracy)}

The overall ability of the KBN-SNPE assay to make the same wild-type or mutation call as the consensus call is defined as follows:

- Overall concordance $=(\#$ of True positives $+\#$ of True negatives) / (\# of True positives + \# of False positives + \# of False negatives + \# True negatives)

Establishing analytical accuracy (using NGS as a tie-breaker assay)

To establish the analytical accuracy of the assay, three sets of samples were analyzed by both the KBN-SNPE assay and Sanger sequencing: cell lines with previously documented mutations in $B R A F$ or $K R A S$; synthetic oligonucleotides containing all mutations detected by 
the KBN-SNPE assay; and a set of 60 FFPE tumor samples (from six different tissues). In summary, the assay was able to make correct calls from all three sample types and the details of the experimental data can be found in the original publication [45].

For FFPE tissue samples, gDNA was isolated from the corresponding 60 FFPE tissue slides, ten samples each of colorectal, ovarian, melanoma, lung (nonsmall cell carcinomas), pancreatic and thyroid cancers. Each sample was subjected to mutation analysis by the KBN-SNPE assay and Sanger sequencing.

\section{Designing SNPE primers to detect certain mutations involving two consecutive nucleotide changes}

The detection of BRAF V600K and V600R requires special probe design since there are two mutations present consecutively in the same codon. In addition to designing oligonucleotide primers/probes, one from sense and one from anti-sense directions, it was necessary to design additional primer/probe in order to cover potential mutant populations. A probe was designed to cover any mutants with A at 1798 nucleotide position to allow detecting mutants with mutations at both 1798 and 1799 nucleotide positions (double-mutation) such as V600R (GTG to AGG) or V600K (GTG to AAG) (see Figure 2 and the original publication). This design was necessary in order to detect the double mutations including the more aggressive V600K BRAF mutation, which has drawn a lot of attention recently for its possible link to tumor metastasis $[66,67]$.

\section{Assay precision \& the limit of detection} (analytical sensitivity)

Assay precision including intra-run and inter-run variation, was done in a traditional way and the detail data can be found in the original publication. As described in the publication, the limit of detection was significantly different for different mutations, ranging from 1.6 to $12 \%$, but since this was determined using cultured cell lines, the behavior and detection sensitivity of these mutations could be quite different from those samples obtained from the FFPE tissue slides. Artifactual mutations such as $\mathrm{C}$ to $\mathrm{T}$ and $\mathrm{C}$ to $\mathrm{A}$ from FFPE tissue samples have been reported in many publications [68-70]. These high background artifactual variant calls interfere with mutation detection calls and will likely affect the detection sensitivity of true mutations. A more practical and perhaps meaningful analytical validation might be to determine the limit of quantification using the triplicate approach, to define the minimum VF for each mutation that could achieve greater than $95 \%$ reproducibility of concordant variant calls. This approach could be very valuable for mutation assay validation in NGS as multiple mutations, including rare and unknown mutations, are impossible to validate individually simply due to the large total number of potential mutations.

\section{Sample analysis in triplicate improves assay performance}

In the experiments described above it was noted that miscalls could occur from time to time depending on the sample quality and the mutant variant frequency. For clinical applications, it would be preferable to reduce these errors, which represent either false positives or false negatives. After careful consideration it was concluded that the error rate of the assay could be reduced by assaying samples in triplicate and taking a 'majority rules' approach. The rules developed for calling KBN-SNPE results are given in Table 5. When samples were assayed in triplicate there was $100 \%$ (42/42) concordance between the KBN-SNPE result and the consensus result (defined by agreement with Sanger or NGS). Samples assayed in triplicate where two or more calls were indeterminate were excluded from calculations for concordance.

The KBN-SNPE assay is a clinical trial assay custom-designed to select patients whose tumors display an activated MAPK pathway. Many analytical validation approaches for this multiplexing mutation assay were modified from the traditional single mutation assay validation strategy, such as gene-level analysis versus sample- or patient-level analysis, however the validation principles remain the same. A triplicate strategy clearly improved the overall performance of this assay where the most challenging clinical FFPE tissue samples were involved and low variant frequency of certain mutations could be encountered.

In general the custom-designed KBN-SNPE assay performance can be summarized below:

- Assay accuracy, as measured by overall concordance to the consensus call for the validation samples, was greater than $94 \%$ at both the patient- and gene-levels for $K R A S, N R A S$ and $B R A F$. The validation samples consisted of ten FFPE sections from six tissue tumor types for a total of 60 samples;

- Intra- and inter-run precision was $97 \%$ at the sample level and greater than $97 \%$ at the gene level;

- Samples assayed in triplicate agreed with the consensus result $100 \%$ of the time at patient and gene levels;

- Concordance was $100 \%$ using gDNA isolated from cell lines with previously defined mutations in $K R A S$ and $B R A F$; 
(A)

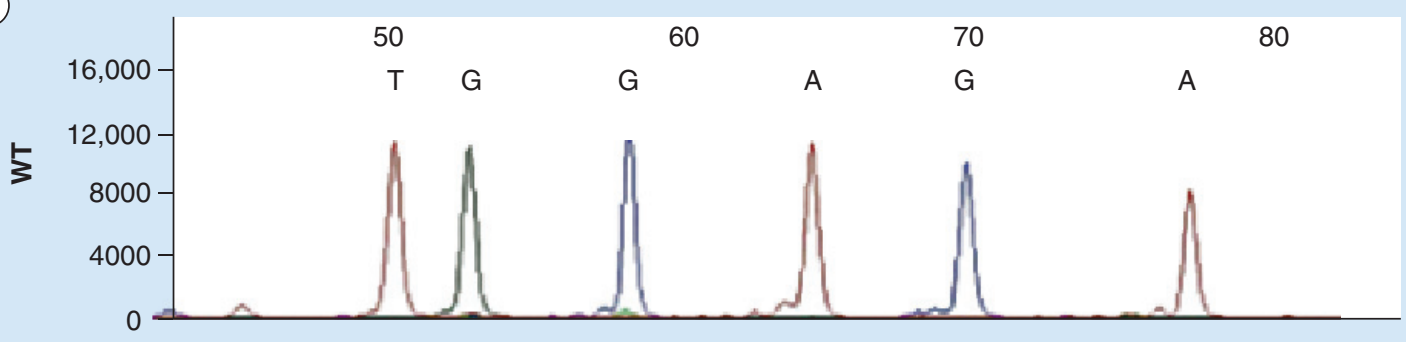

(B)

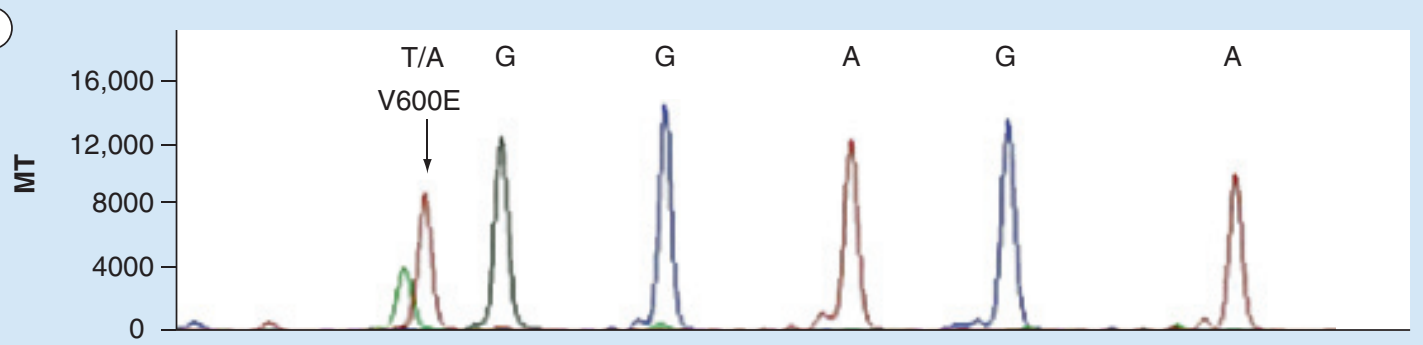

(C)

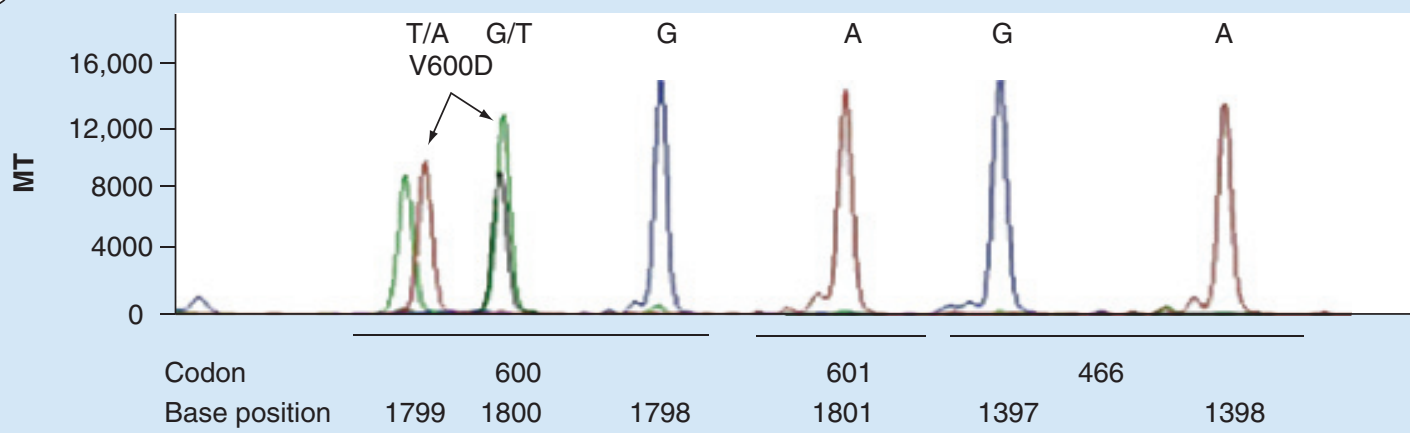

(D)

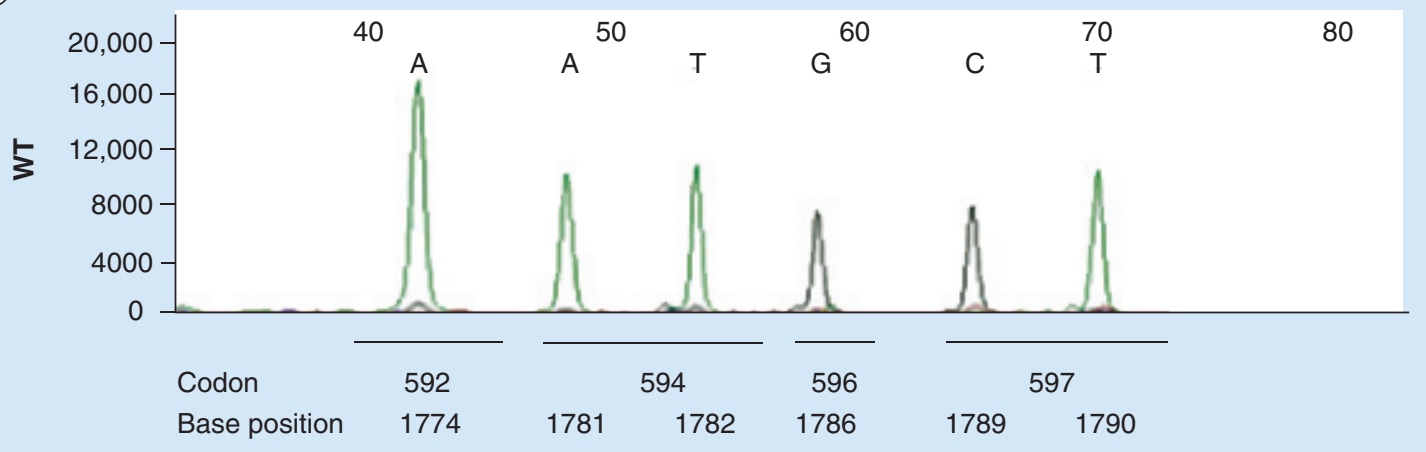

Figure 2. Example of KBN-SNPE results for $B R A F$ probe pools. (A) $B R A F$ probe Pool 1, wild-type control;

(B \& C) Examples of V600E and V600D mutations; (D) BRAF probe Pool 2 wild-type control. Positions of codons and nucleotides are indicated at the bottom of the figure. Nucleotide labels for each peak have been converted to the sense strand.

MT: Mutant; SNPE: Single-nucleotide primer extension; WT: Wild-type.

Reproduced with permission from [45].

- The assay correctly identified all mutations when synthetic oligonucleotides replaced gDNA in the assay;
- The analytical sensitivity of each assay was dependent on the mutation detected and ranged from 1.6 to $12 \%$ mutant DNA against a wild-type background; 
Table 5. Rules for calling KBN-SNPE results at the mutation level when assaying samples in triplicate.

\begin{tabular}{|c|c|c|c|}
\hline \multicolumn{3}{|c|}{ Replicate/call } & \multirow[t]{2}{*}{ Consensus } \\
\hline 1 & 2 & 3 & \\
\hline Wild-type & Wild-type & Wild-type & Wild-type \\
\hline Wild-type & Wild-type & Mutation-1 & Wild-type \\
\hline Wild-type & Mutation-1 & Mutation-1 & Mutation-1 \\
\hline Mutation-1 & Mutation-1 & Mutation-1 & Mutation-1 \\
\hline Wild-type & Wild-type & ND & Wild-type \\
\hline Mutation-1 & Mutation-1 & ND & Mutation-1 \\
\hline Wild-type & ND & ND & Indeterminate-redo \\
\hline ND & ND & ND & Indeterminate-redo \\
\hline Mutation-1 & ND & ND & Indeterminate-redo \\
\hline Wild-type & Mutation-1 & ND & Indeterminate-redo \\
\hline Mutation-1 & Mutation-1 & Mutation-2 & Mutation-1 \\
\hline
\end{tabular}

- The assay efficiency (call rate) for the assay was $>94 \%$ when the claim to have detection sensitivity assay was performed as a single point determination and $100 \%$ when performed in triplicate.

\section{Assay detection sensitivity percentage} reproducibility is crucial to accurate \& consistent diagnosis

Both the Therascreen KRAS and the cobas KRAS and $B R A F$ assays claim to have detection sensitivity of less than 5\% using 100 ng gDNA isolated from FFPE tissue samples with at least $10 \%$ tumor cells on the FFPE tissue slide. For average quality FFPE tissue samples, this translates into approximately 200-400 DNA copies with intact target sequences available for PCR amplification. Therefore, $5 \%$ of a mutant population equals to approximately 10-20 copies, and hence if an aliquot of gDNA isolated from such FFPE tissue slides is used to perform mutation tests, the chance of accurately detecting such a low percentage mutant population is very low [45]. Efforts are being made to establish PCR-based methods of quality control (e.g., determining the number of amplifiable copies of target DNA) to identify samples that meet minimum quality and quantity requirements [71,72]. However, the reality is that if many clinical samples fail to meet these requirements during a clinical trial, it will be essential to find a way to profile these samples otherwise the result may be a failed trial.

For the custom-designed KBN-SNPE multiplexing mutation assay, the detection sensitivity is similar to the FDA-approved KRAS/BRAF assays mentioned above. In our study, to determine assay accuracy, triplicate samples were run using $15 \mathrm{ng}$ of gDNA as starting material for each replicate. Since the KBN-SNPE assay involves fragment separation by capillary electrophoresis and since base calls are based on both migration and color-labeling differences, false positives and false negatives are rare. For samples with low VF mutations, the likely major reason for a discordant call is sampling variation that falls below the detection sensitivity stochastically. Therefore, if only a small number of amplifiable gDNA copies are available as the starting material, a triplicate sample preparation strategy starting from the same gDNA might be necessary to ensure accurate mutation status of clinical samples, especially when low level of mutation detection is critical.

Since most of clinical mutation assays are used for patient treatment guidance and different mutation detection assays have different detection sensitivity, it is very important to recognize that a designation of 'mutation not detected' is very dependent on the assay being used. A mutation status determined to be wildtype for any given samples may not obtain the same mutation status from a different assay. This can be very important clinically, since certain low VF mutations detected in leukemia samples are highly significant in terms of impacting clinical outcome [73].

Furthermore, a recent retrospective analysis of samples from an anti-EGFR clinical study showed that $17 \%$ of the patients called 'mutation not detected' actually had a KRAS mutation detectable by a more sensitive assay [68], some of whom responded to anti-EGFR therapy. Thus, although the correlation between mutation VF and the treatment outcome is yet to be established, these data suggest that some 
patients whose samples contain as much as $10 \%$ mutation may be as responsive as those called wild-type [74]. More and more recent reports have shown that clinical studies using Sanger sequencing for patient enrollment have experienced false-negative calls, in which mutation status was called wild-type, but after retesting the samples were found to harbor mutations using more sensitive FDA-approved tests [35,75]. Many mutations previously believed to be rare, such as BRAF V600K, are now being detected at higher rates due to the improvements of detection sensitivity of new qPCRbased mutation detection assays and very sensitive and widely used NGS assays, which are reported to permit detection of mutant allele frequency at low single-digit percentage levels $[76,77]$. These examples illustrate that the definition of wild-type ('mutation not detected') is assay-dependent. While this has always been true, this has important implications for the development of future mutation detection assays, as discussed in the following sections.

\section{NGS assay design \& special considerations for querying MAPK pathway activation}

As mentioned above, if the location of a possible mutation is unknown, then a general sequencing approach is necessary; this includes next generation sequencing. NGS is also known as massively parallel sequencing, as millions of sequencing reads are done in the same chip or flow cell in each sequencing run. Reviews of this exciting new technology and its application in clinical research can be found in numerous journals, and a selective set of articles is included here [78-82]. The recent FDA clearance of the Illumina MiSeqDx platform [83] is likely to encourage clinical application of this novel technology. If NGS is to be used in querying MAPK pathway activation, one can easily design a MAPK pathway-focused gene panel, and all targeted sequence regions of each gene could be fully covered with both known and unknown mutations, profiled in detail. Previous assay design strategies querying MAPK pathway activation were focused on the interrogation of hotspot mutations of each gene. However, as mentioned above, recent data suggest that the omission of rare or unknown mutations from the common mutation assays may cause the misdiagnosis of many patients and the underestimation of the efficacy of some investigational drugs, by including patients that should have been excluded according to the study inclusion criteria. Therefore, it may be beneficial to cover the entire gene of interest in a focused NGS gene panel. Recently, Amgen and Illumina announced a companion diagnostics agreement in which Illumina will develop a multi-gene, NGSbased test as a companion diagnostic for Vectibix (panitumumab), a metastatic colorectal cancer drug, to more comprehensively detect the MAPK pathway mutations [84]. Analytical validation of such an NGS panel for clinical application may be very challenging since many performance metrics for more traditional mutation assays will need to be modified. First, many traditional mutation assays are designed to detect one mutation at a time. The degree of complexity for detecting well-defined mutations is much smaller than that for an NGS mutation detection assay where hundreds, if not thousands, of variant calls could be generated from one sample. How to calculate this type of multivariant repeatability or reproducibility is a challenge, not to mention that the assessment of accuracy for hundreds of variant calls may render the task extremely difficult and time consuming, if not entirely impossible. Therefore, novel approaches may need to be devised in order to optimally utilize this powerful technology. For example, using a triplicate approach and a concordance calculator to sort out concordant and discordant variant calls could be very valuable [85]. Furthermore, using a triplicate strategy to optimize quality control filters, including VF, coverage, quality scores, and other bioinformatics filters, to reduce false positive and false negative rates could potentially be beneficial for determining/defining the assay specificity, precision and limit of detection [85]. Another similar approach used quality metrics to rank order the target variants and plot the accumulation rate for both concordant and discordant variants with decreasing score stringency [86]. A more detailed discussion is outside of the scope of this review.

\section{Discussion \& future perspective}

In this article, many genomics assays that could be employed to probe MAPK pathway activation were reviewed. A recent example of developing and analytically validating a custom-designed mutation assay to detect 35 mutations of interest in the KRAS, BRAF and $N R A S$ genes in FFPE tumor tissue for patient stratification in clinical trials, was provided. In this multiplexed mutation assay, several novel analytical validation approaches were taken to reduce the likelihood of false-positive calls, which are an issue associated with clinical FFPE tissue [63]. Recently, several publications showed that many mutations in COSMIC were reported only once, suggesting a strong possibility of a false-positive call $[69,87]$. This will likely trace back to the validity of many analytically validated clinical mutation assays. Whether the mutation assays used in the clinical sample testing were properly validated or not is debatable [88,89]. Nonetheless, the reproducibility of rare or unknown mutations detected from these assays should always be addressed. 
One frequently encountered issue during clinical sample testing is the high sample failure rate resulting from the samples' inability to meet minimum quality or quantity requirements. Typically, a biomarker strategy starts with acquiring high quality preclinical and clinical biosamples in order to facilitate biomarker discovery. However, from a clinical assay development and validation point of view, this approach may have unintended consequences, since it may lead to assays that are difficult to validate clinically with actual clinical samples, which normally have lower quality. Without improving sample collection and storage issues at the clinical sites, the use of high quality samples for biomarker discovery and development may make it difficult to reproduce a preclinically observed biomarker effect in a clinical setting. Therefore, in the biomarker discovery-development stage, preclinical samples with quality that mimics that of poorer quality clinical samples should be evaluated. This could be done by using preclinical samples that went through a controlled degradation/fragmentation process, for the preclinical biomarker validation. The analytical validation plan should also consider including a section to address the limitation of the assay when lower quality samples are encountered, therefore allowing the clinical team to more accurately calculate or predict the enrollment rate. It would be a disastrous situation if a clinical trial were terminated not because the lack of drug efficacy, but instead due to a poor enrollment rate that was not anticipated by the clinical team.

Validating the reproducibility of any specific mutation does not guarantee the validity of different mutations. The KBN-SNPE mutation assay described in this review article showed that different mutations have different detection sensitivity and any mutations detected close to their detection sensitivity will be unlikely to be reproducible. Therefore, the KBNSNPE assay employed a triplicate strategy to address this issue which resulted in the improvement of assay performance and reduced false positives and false negatives to almost non-existence. The fundamental issue for FFPE tissue samples is the degree of fragmentation of the nucleic acids and the limited quantity available from each FFPE tissue slide. In other words, when the mutation assay uses only a small amount of gDNA, the limited number of copies of DNA available for PCR amplification shifts the focus of the question to sampling variation and the fundamental principle of probability. If a limited copy number of DNA is used, simple sampling variation can easily cause a two to threefold variation in variants with low $\mathrm{VF}$, and further variation will be introduced if additional manipulations throughout the library preparation or entire protocol, including PCR amplification, are needed. With a small number of DNA copies available, improper or insufficient sample and reagent mixing during each step of the assay could also introduce large variation. Additional considerations from a thermodynamics and kinetics point of view, such as proper hybridization to the specific target sequence, and the competition among minor and major alleles for the primers/probes or even ligation adaptors, could also play critical roles. Many analytical validation strategies for mutation assays do not use the true clinically mimetic samples,

\section{Executive summary}

Brief overview of mutation assays that detect MAPK pathway gene activation

- Type of MAPK pathway activation mutation assays can be classified into two broad categories: those that detect mutations in specific, limited nucleotide locations, and those that take a general sequencing approach such as Sanger sequencing or pyrosequencing.

- Many clinical considerations based on clinical objectives are important to the selection of a specific assay for the clinical application.

- Additional assay development considerations include tissue types, sample collection and preservation methods, clinical objectives, turn-around time, and acceptable false-positive/false-negative rates.

Novel features of a custom-designed multiplexed mutation assay

- The KRAS, BRAF, NRAS SNPE (KBN-SNPE) mutation detection assay was designed to support an ongoing clinical trial for prospective patient enrollment.

- The analytical validation process of KBN-SNPE assay involved a triplicate strategy and relied on several novel approaches to redefine and calculate sensitivity, specificity and accuracy.

- The KBN-SNPE mutation assay described in this review article showed different mutations have different detection sensitivity and any mutations detected close to their detection sensitivity are unlikely to be reproducible.

\section{Future perspective}

- In the biomarker discovery-development stage, preclinical samples with quality that mimics that of poorer quality clinical samples should be evaluated.

- The triplicate strategy should be considered to improve assay reproducibility for any mutation assays including next generation sequencing, especially for those variant calls around detection sensitivity. 
which usually have poorer average quality than those procured from the commercial tissue source. Even if the clinically representative average-quality samples are used for the analytical validation, those clinical samples with below-average quality might have irrational results that were not covered by the validation strategy. Hence, accepting those results based on a successful analytical validation will still lead to the identification of non-reproducible mutations which could be mostly eliminated by implementing a triplicate strategy. Of course, cost is always a concern for running singlet mutation assay versus triplicate mutation assay. However, based on clinical experience, samples usually arrive in batches and due to TAT requirements they are likely to be processed a few samples at a time. Either using a 96-well plate assay for PCR-based mutation detection or a chip or flow cell for NGS assay, running triplicate (starting from the same gDNA stock solution) will be unlikely to increase the cost threefold. Nonetheless, cost should not be the reason for compromising the clinical assay reproducibility.

As discussed in the previous NGS assay design and considerations section, it is likely that NGS will be used more widely in querying MAPK pathway activation

\section{References}

1 Yokota T. Are KRAS/BRAF mutations potent prognostic and/or predictive biomarkers in colorectal cancers? Anticancer Agents Med. Chem. 12(2), 163-171 (2012).

2 Messersmith WA, Ahnen DJ. Targeting EGFR in colorectal cancer. N. Engl. J. Med. 359(17), 1834-1836 (2008).

3 Morris EJ, Jha S, Restaino CR et al. Discovery of a novel ERK inhibitor with activity in models of acquired resistance to BRAF and MEK inhibitors. Cancer Discov. 3(7), 742-750 (2013).

4 Downward J. Targeting $R A S$ signaling pathways in cancer therapy. Nat. Rev. Cancer 3(1), 11-22 (2003).

5 Pearson G, Robinson F, Beers Gibson T, Xu B-E, Karandikar M et al. Mitogen-activated protein (MAP) kinase pathways: regulation and physiological functions. Endocr. Rev. 22(2), 153-183 (2001).

6 Fecher LA, Amaravadi RK, Flaherty KT. The MAPK pathway in melanoma. Curr. Opin Oncol. 20 (2), 183-189 (2008).

7 Dellavalle RP, Nicholas MK, Schilling LM. Melanoma chemoprevention: a role for statins or fibrates? Am. J. Ther. 10(3), 203-210 (2003).

8 Kelland LR. Farnesyl transferase inhibitors in the treatment of breast cancer. Expert Opin. Emerg. Drugs 12(3), 413-421 (2003).

9 Rose WC, Lee FY, Fairchild CR et al. Preclinical anti-tumor activity of BMS-214662, a highly apoptotic and novel farnesyltransferase inhibitor. Cancer Res. 61(20), 7507-7517 (2001). in the future. Since NGS can cover both known and unknown mutations, and use of the less sensitive mutation assays may cause the misdiagnosis of many patients, it may be beneficial to cover the entire gene of interest using a focused NGS gene panel. The above mentioned Amgen and Illumina companion diagnostics agreement should pave the way for querying the MAPK pathway activation in a more comprehensive manner.

\section{Acknowledgements}

The authors would like to thank S Galuska of LabCorp for his contribution in the KBN-SNPE assay development and validation work.

\section{Financial \& competing interests disclosure}

Both authors are full time employees of Merck and therefore receive salary and stocks. The authors have no other relevant affiliations or financial involvement with any organization or entity with a financial interest in or financial conflict with the subject matter or materials discussed in the manuscript apart from those disclosed.

No writing assistance was utilized in the production of this manuscript.

10 Rose WC, Long BH, Fairchild CR et al. Preclinical pharmacology of BMS-275183, an orally active taxane. Clin. Cancer Res. 7(7), 2016-2021 (2001).

11 Dinsmore CJ, Bogusky MJ, Culberson JC et al. Conformational restriction of flexible ligands guided by the transferred noe experiment: potent macrocyclic inhibitors of farnesyltransferase. J. Am. Chem. Soc. 123(9), 2107-2108 (2001).

12 Lorusso PM, Adjei AA, Varterasian M et al. Phase I and pharmacodynamic study of the oral MEK inhibitor CI1040 in patients with advanced malignancies. J. Clin. Oncol. 23(23), 5281-5293 (2005).

13 Solit DB, Garraway LA, Pratilas CA et al. BRAF mutation predicts sensitivity to MEK inhibition. Nature 439(7074), 358-362 (2006).

14 LoRusso PM, Krishnamurthi SS, Rinehart JJ et al. Phase I pharmacokinetic and pharmacodynamics study of the oral MAPK/ERK kinase inhibitor PD-0325901 in patients with advanced cancers. Clin. Cancer Res. 16(6), 1924-1937 (2010).

15 Iverson C, Larson G, Lai C et al. RDEA119/BAY 869766: a potent, selective, allosteric inhibitor of MEK1/2 for the treatment of cancer. Cancer Res. 69 (17), 6839-6847 (2009).

16 Daouti S, Higgins B, Kolinsky K et al. Preclinical in vivo evaluation of efficacy, pharmacokinetics, and pharmacodynamics of a novel MEK1/2 kinase inhibitor RO5068760 in multiple tumor models. Mol. Cancer Ther. 9(1), 134-144 (2010).

17 Huang T, Karsy M, Zhuge J et al. B-Raf and the inhibitors: From bench to bedside. J. Hematol. Oncol. 6, 30 (2013). 
18 Flaherty KT, Infante JR, Daud A et al. Combined BRAF and MEK Inhibition in Melanoma with BRAF V600 Mutations. N. Engl. J. Med. 367(18), 1694-1703 (2012).

19 Garber K. Melanoma drug vindicates targeted approach. Science 326(5960), 1619 (2009).

20 Poulikakos PI, Zhang C, Bollag G et al. RAF inhibitors transactivate RAF dimers and ERK signalling in cells with wild-type BRAF. Nature 464(7287), 427-430 (2010).

21 Wilhelm SM, Carter C, Tang L et al. BAY 43-9006 exhibits broad spectrum oral antitumor activity and targets the RAF/ MEK/ERK pathway and receptor tyrosine kinases involved in tumor progression and angiogenesis. Cancer Res. 64(19), 7099-7109 (2004)

22 Eisen T, Ahmad T, Flaherty KT et al. Sorafenib in advanced melanoma: a Phase II randomized discontinuation trial analysis. Br. J. Cancer 95(5), 581-586 (2006).

23 Joseph EW, Pratilas CA, Poulikakos PI et al. The RAF inhibitor PLX4032 inhibits ERK signaling and tumor cell proliferation in a V600E BRAF-selective manner. Proc. Natl Acad. Sci. USA 107(33), 14903-11498 (2010).

24 Flaherty KT, Puzanov I, Kim KB et al. Inhibition of mutated, activated BRAF in metastatic melanoma. N. Engl. J. Med. 363(9), 809-819 (2010).

25 Mordant P, Loriot $\mathrm{Y}$, Leteur $\mathrm{C}$ et al. Dependence on phosphoinositide 3-kinase and RAS-RAF pathways drive the activity of RAF265, a novel RAF/VEGFR2 inhibitor, and RAD001 (Everolimus) in combination. Mol. Cancer Ther. 9(2), 358-368 (2010).

26 Kefford R, Arkenau H, Brown MO et al. Phase I/II study of GSK2118436, a selective inhibitor of oncogenic mutant BRAF kinase, in patients with metastatic melanoma and other solid tumors. J. Clin. Oncol. 28, 15 (2010).

27 Arkenau HT, Kefford R, Long GV. Targeting BRAF for patients with melanoma. Br. J. Cancer 104(3), 392-398 (2011).

28 Smalley KS. A pivotal role for ERK in the oncogenic behavior of malignant melanoma? Int. J. Cancer 104(5), 527-532 (2003).

29 Roberts PJ, Der CJ. Targeting the Raf-MEK-ERK mitogenactivated protein kinase cascade for the treatment of cancer. Oncogene 26(22), 3291-3310 (2007)

30 Wistuba II, Gazdar AF, Minna JD. Molecular genetics of small cell lung carcinoma. Semin. Oncol. 28(2Suppl4), 3-13 (2001).

31 Davies H, Bignell GR, Cox C et al. Mutations of the BRAF gene in human cancer. Nature 417(6892), 949-954 (2002).

32 Kohno M, Pouyssegur J. Targeting the ERK signaling pathway in cancer therapy. Ann. Med. 38(3), 200-211 (2006).

33 Brose MS, Volpe P, Feldman M et al. BRAF and RAS mutations in human lung cancer and melanoma. Cancer Res. 62(23), 6997-7000 (2002).

34 Pratilas CA, Solit DB. Therapeutic strategies for targeting $B R A F$ in human cancer. Rev. Recent Clin. Trials 2(2), 121-134 (2007).

35 Andre T, Blons $\mathrm{H}$, Mabro $\mathrm{M}$ et al. Pantiumumab combined with irinotecan for patients with $K R A S$ wild- type metastatic colorectal cancer refractory to standard chemotherapy: a GERCOR efficacy, tolerance, and translational molecular study. Ann. Oncol. 24(2), 412-419 (2012).

36 DeRook W, Claes B, Bernasconi D et al. Effects of KRAS, $B R A F, N R A S$ and PIK3CA mutations on the efficacy of cetuximab plus chemotherapy in chemotherapy-refractory metastatic colorectal cancer: a retrospective consortium analysis. Lancet Oncol. 11(8), 753-6762 (2010).

37 Douillard JY, Oliner KS, Siena S et al. PanitumumabFOLFOX4 treatment and RAS mutations in colorectal cancer. N. Engl. J. Med. 369(11), 1023-1034 (2013).

38 Goodman A. Call for expanded genetic profiling in colorectal cancer. ASCO 4, 20 (2013).

39 Tougeron D, Lecomte T, Pages JC et al. Effect of lowfrequency $K R A S$ mutations on the response to anti-EGFR therapy in metastatic colorectal cancer. Ann. Oncol. 24(5), 1267-1273 (2013).

40 Mao C, Huang YF, Yang ZY et al. KRAS p.G13D mutation and codon 12 mutations are not created equal in predicting clinical outcomes of cetuximab in metastatic colorectal cancer: a systematic review and meta-analysis. Cancer 119(4), 714-721 (2013).

41 Rollins $\mathrm{G}$. The promise and power of next generation sequencing. AACC 38, 4 (2012)

42 Nyren P. The history of pyrosequencing. Methods Mol. Biol. 373, 1-14 (2007).

43 Lurkin I, Stoehr R, Hurst CD, van Tilborg AAG, Knowles MA et al. Two multiplex assays that simultaneously identify 22 possible mutation sites in the KRAS, BRAF, NRAS and PIK3CA genes. PLoS ONE 5(1), e8802 (2010).

44 Dias-Santagata D, Akhavanfard S, David SS et al. Rapid targeted mutational analysis of human tumours: a clinical platform to guide personalized cancer medicine. $E M B O$ Mol. Med. 2(5), 146-158 (2010).

45 Chang KCN, Galuska S, Weiner R et al. Development and validation of a clinical trial patient stratification assay that interrogates 27 mutation sites in MAPK pathway genes. PLos ONE 8(8), e72239 (2013).

46 Kang SY, Ahn S, Lee SM et al. Shifted termination assay (STA) fragment analysis to detect BRAF V600 mutations in papillary thyroid carcinomas. Diagn. Pathol. 8(1), 121 (2013).

47 Sarasqueta AF, Moerland E, de Bruyne $\mathrm{H}$ et al. SNaPshot and StripAssay as valuable alternatives to direct sequencing for $K R A S$ mutation detection in colon cancer routine diagnostics. J. Mol. Diagn. 13(2), 199-205 (2011).

48 Machnicki MM, Glodkowska-Mrowka E, Lewandowski T et al. ARMS-PCR for detection of BRAF V600E hotspot mutation in comparison with real-time PCR-based techniques. Acta Biochim. Pol. 60 (1), 57-64 (2013).

49 McGuigan FE, Ralston SH. Single nucleotide polymorphism detection: allelic discrimination using TaqMan. Psychiatr. Genet. 12(3), 133-136 (2002).

50 Thomas RK, Baker AC, DeBiasi RM et al. Highthroughput oncogene mutation profiling in human cancer. Nat. Genet. 39(3), 347-351 (2007). 
Cobas KRAS Mutation Kit.

www.molecular.roche.com

Test.

www.molecular.roche.com

53 www.transgenomic.com

54 Therascreen KRAS RGQ PCR Kit. www.fda.gov

55 bioMerieux THxID ${ }^{\text {TM}}$-BRAF Kit.

www.biomerieux-usa.com

56 Loboda A, Nebozhyn M, Klinghoffer R et al. A gene expression signature of RAS pathway dependence predicts response to PI3K and RAS pathway inhibitors and expands the population of RAS pathway activated tumors. $B M C$ Med. Genomics 3, 26 (2013).

57 Marton MJ, Weiner R. Practical guidance for implementing predictive biomarkers into early phase clinical studies. Biomed. Res. Int. 2013, 891391 (2013).

58 A study of the safety, tolerability, and efficacy of mk-8353 in participants with advanced solid tumors. www.clinicaltrials.gov

59 Davies H, Bignell GR, Cox C, Stephens P et al. Mutations of the BRAF gene in human cancer. Nature 417(6892), 949-954 (2002).

60 Flaherty KT, McArthur G. BRAF, a target in melanoma: implications for solid tumor drug development. Cancer 116(21), 4902-4913 (2010).

61 Vakiani E, Solit DB. KRAS and BRAF: drug targets and predictive biomarkers. J. Pathol. 223(2), 219-229 (2011).

62 Riely GJ, Marks J, Pao W. KRAS mutations in non-small cell lung cancer. Proc. Am. Thorac. Soc. 6(2), 201-205 (2009).

63 Lau KS, Haigis KM. Non-redundancy within the RAS oncogene family: insights into mutational disparities in cancer. Mol. Cells 28(4), 315-320 (2009).

64 Janakiraman M. Vakiani E, Zeng Z et al. Genomic and biological characterization of exon 4 KRAS mutations in human cancer. Cancer Res. 70 (14), 5901-5911 (2010).

65 Dias-Santagata D, Lam Q, Vernovsky K et al. BRAF V600E mutations are common in pleomorphic xanthoastrocytoma: Diagnostic and therapeutic implications. PLoS ONE 6(3), e17948 (2011).

66 Menzies AM, Haydu LE, Visintin L et al. Distinguishing clinicopathologic features of patients with V600E and V600K BRAF-mutant metastatic melanoma. Clin. Cancer Res. 18(12), 3242-3249 (2012).

67 Jewell R, Chambers P, Harland M et al. Clinicopathologic features of V600E and V600K melanoma - letter. Clin. Cancer Res. 18(24), 6792-6793 (2012).

68 Costello M, Pugh TJ, Fennell TJ et al. Discovery and characterization of artifactual mutations in deep coverage targeted capture sequencing data due to oxidative DNA damage during sample preparation. Nucleic Acids Res. 41(6), e67 (2013)

69 Do H, Dobrovic A. Dramatic reduction of sequence artefacts from DNA isolated from formalin-fixed cancer biopsies by treatment with uracil-DNA glycosylase. Oncotarget 3(5), 546-558 (2012).

70 Quach N, Goodman MF, Shibata D. In vitro mutation artifacts after formalin fixation and error prone translesion synthesis during PCR. BMC Clin. Pathol. 4(1), 14 (2008).

71 Duenwald S, Zhou M, Wang Y et al. Development of a microarray platform for FFPET profiling: application to the classification of human tumors. J. Transl. Med. 7, 65 (2009)

72 Sah S, Chen L, Houghton J et al. Functional DNA quantification guides accurate next-generation sequencing mutation detection in formalin-fixed, paraffin-embedded tumor biopsies. Genome Med. 5(8), 77 (2013).

73 Parker WT, Ho M, Scott HS et al. Poor response to second-line kinase inhibitors in chronic myeloid resistance profile leukemia patients with multiple low-level mutations irrespective of their resistance profile. Blood 119(10), 2234-2238 (2012).

74 Tougeron D, Lecomte T, Pagès JC et al. Effect of lowfrequency KRAS mutations on the response to anti-EGFR therapy in metastatic colorectal cancer. Ann. Oncol. 24(5), 1267-1273 (2013)

75 TP53 Status may predict benefit from cetuximab in locally advanced rectal cancer. www.ascopost.com

76 Schmitt MW, Kennedy SR, Salk JJ et al. Detection of ultrarare mutations by next-generation sequencing. Proc. Natl Acad. Sci. USA 109(36), 14508-14513 (2012).

77 Flaherty P, Natsoulis G, Muralidharan O et al. Ultrasensitive detection of rare mutations using next-generation targeted resequencing. Nucleic Acids Res. 40 (1), e2 (2012).

78 Shendure J, Ji H. Next-generation DNA sequencing. Nat. Biotechnol. 26(10), 1135-1145 (2008).

79 Hanahan D, Weinberg RA. Hallmarks of cancer: the next generation. Cell 144(5), 646-674 (2011).

80 Roukos DH. Next-generation, genome sequencing-based biomarkers: concerns and challenges for medical practice. Biomark. Med. 4(4), 583-586 (2010).

81 The Cancer Genome Atlas Network. Comprehensive molecular characterization of human colon and rectal cancer. Nature 487(7407), 330-337 (2012).

82 Pennisi E. Genome sequencing. Search for pore-fection. Science 336(6081), 534-537 (2012).

83 Pant S, Weiner R, Marton JM. Navigating the rapids: the development of NGS-based clinical trial assays and companion diagnostics. Front Oncol. 4, 78 (2014).

84 Illumina and Amgen Collaborate for Cancer Companion Diagnostic Test. www.nasprx.org

85 Chang KCN, Zhao Y, Kang J et al. Validation of next generation sequencing cancer panels for clinical somatic mutation profiling - identification of source of variations and artifacts using FFPE tissues. J. Clin. Oncol. 32(Suppl.), Abstract e22180 (2014).

86 Robasky K, Lewis NE, Church GM. The role of replicates for error mitigation in next-generation sequencing. Nature Perspectives 15(1), 56-62 (2014). 
87 Lawrence MS, Stojanov P, Polak P et al. Mutational heterogeneity in cancer and the search for new cancerassociated genes. Nature 499 (7457), 214-218 (2013).

88 Dubbink HJ, Deans ZC, Tops BBJ et al. Next generation diagnostic molecular pathology: critical appraisal of quality assurance in Europe. Mol. Oncol. 8(4), 830-839.
89 Salto-Tellez M, de Castro DG. Next generation sequencing: a change of paradigm in molecular diagnostic validation. J. Pathol. doi:10.1002path.4251 (2013) (Epub ahead of print). 